siderable, that the patient dreads the process of eracuating the bladder. Painful micturition, extending over a considerable time, in a middle-aged woman, should lead us to suspect the presence of this affection. Examination of the meatus would then be necessary.

Another class of cases of painful micturition are those in which the bladder and urethra are unaffected, but, the ostium vagince being in an inflamed condition, the passage of urine is productive of pain from the contact of the latter with the inflamed surface. Certain forms of leucorrhoea are associated with painful micturition, in consequence of the existence of this inflammators condition of the outlet of the vagina. When the upper and inner part of the thighs are excoriated by contact with irritating discharges of a cancerous or other nature, the patient will lead us to infer that there is painful micturition; the pain arising in the latter case also from contact of the urine with a raw inflamed surface. The immediate neighbourhood of the outlet of the urinary meatus may be inflamed as the result of masturbation, as was the case in a young lady recently under my observation. The painful micturition was here the symptom most prominently attracting attention.

Alterations in the position of the uterus, thereby drawing the urethra out of its place, alterations of the bladder itself, or tumours of adjacent organs, may produce difficulty in micturition, as already pointed out. The difficulty is generally accompanied with more or less pain; but the pain is not, as a rule, the prominent symptom, though it may be so in a few exceptional cases. With a little care in cross-examination, it may generally be made out whether the pain or the difficulty came first in order of sequence, and this point is of importance in reference to the diagnosis.

Female Convicts. Dr. Guy, in his report, gives an account of one of these convicts, who was only subdued by a long course of firm treatment. She had the strength of a man and the agility of a monkey. Sooner or later she burst all the strait jackets in use on the women's side of the prison, repeatedly tore down with her teeth the strong canvass of the padded cell, and even broke down the ceiling. There is a class of women met with in the convict prisons so hardened that the chuplain of Brixton says they are almost "a wonder"; and he can only exclaim, "Though thou shouldst bray a fool in a mortar, yet will not his foolish. ness depart from him."

Sir J. McGrigor. Before the siege of Badajoz the medical officers were never nuticed in the dispatches. However much they risked their lives, their services were known only to their comrades. After the siege, finding Lord Wellington in excellent spirits, he said"I trust, my Lord, you are satisfied that the medical officers during last night did their duty, as well as the military officers, and that you will receive my testimony that they discharged their arduous and laborious duties most zealously, and often under circumstances of personal danger, of which they were regardless," He replied that " he himself had witnessed it." I then added, no thing could more gratify those officers-nothing could be a greater incentive to their exertions on future occasions, than his noticing them in his public dispatches. $\mathrm{He}$ asked, "Is that usual ?" My reply was, "It would be of the most essential service." Although the dispatch was finished, "something about the doctors" was added. (Med. Record.)

\section{clinical deetures}

DELIVERED hT

\section{CHARING CROSS HOSPITAL.}

\author{
BY
}

HYDE SALTER, M.D., F.R.S.,

FELLOW OF THE ROYAL COLLEGE OF PHYSICIANS; LECTURER ON PHYSIOLOGY AND PATHOLOGY AT CHARING CROSS HOSPITAL MEDICAL SCHOOL; AND ASSISTANTPHYSICIAN TO THE hOSPITAL.

Lecture I. (Concluded.)-Regurgitant Aortic Disease.

Aortic Regurgitant Pulse: its Mechanism. The Three Circumstances conspiring to downuard Displacement of Heart's Dulness. Cardiac Sputum. Diagnostic Value of Age in the Etiology of Organic Heart-Disease. Treatment.

Is prosecuting our inquiry into our patient's symptoms beyond what superficially manifests itself, the first thing that would strike us would be his pulse. Now, this pulse is utterly peculiar; it is diagnostic of aortic regurgitation; in no other state whatever could it occur ; it points to that one thing alone ; and any one of you might on the strength of it, and without making any examination of the heart, safely diagnose the valvular lesion. When I first listened to this patient's chest, the only sound that I heard was at the base, with the first sound (systolic aortic), and I therefore pronounced the disease to be aortic obstruction; but, the moment I saw the pulse, I knew there must be aortic regurgitation, and, on listening again, plainly heard, at the same situation, a bruit with the second sound (diastolic aortic); and we have heard it ever since, much more plainly than the systolic; and how it was it escaped me at first, I am at a loss to explain. I need not tell you that the peculiar character of the pulse to which I refer is a conspicuous leaping jerk, not only perceptible to the touch, but visible to the eye ; carotids, temporals, brachials, radials, tibials-in fact, all the arteries wherever superficial, and where not ordinarily visible, are plainly seen leaping forth at each beat of the heart; and the pulse may be counted in all of them, as well by the eye as by the touch. The arterial system seems alive; and, whenever the arteries are tortuous, a peculiar sudden writhe is visible, like a centipede when touched. This pulse has been called a "jerking" pulse ; I think a "leaping" pulse would perhaps be a better name. It was called by Dr. Todd a "locomotive" pulse-an injudicious name, I think ; for locomotive is just what it is not : it is motive, but not locomotive.

The way in which it is produced by the lesion which it implies is sufficiently clear. When the aortic valves are sound, the complete and instantaneous closure of the aortic orifice maintains the tension of the arterial system which the additional volume of blood sent into it by the systole has just carried to its maximum. The systole makes the arterial system tense, the valves keep it tense, and therefore the next systole finds it tense ; but where, from insufficiency of the aortic valves, the blood largely refluxes into the ventricle, the tension of the arteries is suddenly lost, their tendency to contract on their contents is unresisted, and they instantly collapse. The state of the arterial system at systole 
and diastole is, in the one case, full-kept full; full -kept full: in the other it is, full-empty; fullempty. It is this alternation of a condition of fulness and emptiness, instead of the maintenance of a nearly uniform fulness, that mainly contributes to this jumping character of the pulse. I am accustomed to compare the natural pulse to the dead beat of a chronometer, and the regurgitant pulse to the beat of the second-hand of an ordinary clock. In the one, for every second moved the hand moves forward a second, and then holds its own : in the other, there is a back stroke of as much as half a second; so that for every beat the second-hand moves forward a second and a half, and back half a second. Which looks the most "lively" and mobile, all will know who have watched the two methods of beat: in fact, one goes over just twice as much ground as the other in the same amount of progress. The ana$\operatorname{logy}$ of these two beats to the natural and jerking pulse is, I think, very close. Moreover, a ventricle emptying itself into a collapsed arterial system would probably do so in a more jerky and sudden way, than if driving its contents into an already tense and preoccupied aorta. Moreover, the more tense the arterial wall, the greater and the more sustained is its reaction upon the contained blood, and the more complete that conversion of the systolic pumping into the even flow characteristic of the circulation in the smaller ressels, which it is the purpose and effect of this elastic reaction to produce. The sudden loss of arterial tension on the reflux of the blood into the ventricle must almost destroy this conversion of the intermittent systole into the sustained vis a tergo of the more distant arterial streans ; and thus we see, in these cases, the smallest arteries beating with almost as much jerk as the largest. Moreover, at each diastole, the blood in the arteries must, comparatively, come almost to a standstill; therefore the next jerk finds a too stationary blood in front of it-a blood, therefore, more than usually antagonising the onward flow of the fresh instalment from the ventricle. Now, we know that the force of the ventricle is partly spent in driving the blood onwards ; and partly, in consequence of the resistance that it experiences in this direction, that it acts laterally on the arterial walls, and distends the arteries. The greater, therefore, the resistance that it experiences in front, the more it is spent laterally.

In all these ways, aortic regurgitation conspires to produce the excessive arterial pulsation that presents so remarkable an appearance in our patient.

On examining the heart, we find, besides the sounds I have described, that it is leating with a too strong and heary impulse, that the apex beat is too low, and that the heart altogether is greatly displaced downwards. Now, I am sure it is a mistake to regard this downward displacement as due only to hypertrophy. Three causes conspire to produce it :-one, the hypertrophy of the organ; another, the dyspnocal intlation of the lungs; and another, the flattening of the diaphragm, which always exists where there is an abiding source of dyspnœe, where there is an unsatisfied and unsatisfiable demand of the conditions of respiratory balance. This descent and flattening of the diaphragm is, in fact, a part of that chest distension which immediately supervenes whenever there is a condition of respiratory arrears; it is the instinctive and direct effort to set things right, and restore the balance, by getting more air. If you examine the heart of a person panting from exertion, you will find it beating considerably lower than usual ; the moment a paroxysm of asthma comes on down goes the heart from this descent of the diaphragm, and may be plainly felt beating in the scrobiculus; in fact, in all dyspnoas this is the case, and the dyspnoea of heart-disease is not an exception. For although, in heart-disease, the disturbance being on the side of blood-supply, and not on that of air-supply, the introduction of an additional quantity of air does not better the state of things; it, nevertheless, instinctively occurs. It is, therefore, a great mistake to imagine that the amount of hypertrophy may be measured by the amount of downward displacement of the cardiac dulness. A striking confirmation of this is shown in the fact that while, in cases of undoubted hypertrophy, there is great downward displacement of the heart's dulness, there is often no upward extension of it at all ; nay, that it will not extend as high as usual. The fact is, that with the dyspnceal chestdistension there is dyspnœal lung-inflation, the inflated left lung overlaps the heart in an excessive degree above and to the left, and thus we get, to a certain extent, the same state of things as in emphysema. The unduly expanded left lung may also be said to thrust the heart downwards and to the right. In a temporary source of dyspnœa, as asthma or violent exertion, this state of things is essentially temporary; and as soon as the breathlessness is over the heart returns to its normal position, and its dulness to its normal limits. But in such a case as our patient's, this condition is permanent, and we see it in him very strongly marked. The dulness involves the ensiform cartilage and the sternal extremity of the eighth, and even the ninth, rib; while, above, the cartilage of the fifth and the corresponding portion of the sternum is resonant.

We must, therefore, be on our guard, on the one hand, against imagining that the whole of the downward extension of the heart's dulness is to be explained by the hypertrophy; and, on the other hand, that because there is no upward extension of dulness, or even less than usual, there is, therefore, no hypertrophy at all ; for you may have considerable downward extension of dulness, and yet there shall be no hypertrophy; you may have no upward extension, and yet hypertrophy shall exist.

And let me take this opportunity of earnestly impressing upon you the importance of bearing in mind this axiom (if I may so call it) in auscultation, that any source of dyspnoa induces a condition of hyperdistension of the chest, temporary if the source of dyspnoca is temporary, abiding if abiding. Unless you bear this in mind, you will be in constant danger of drawing erroneous conclusions as to the existence of emphysema, heart-disease, and various other conditions. I the more earnestly impress it upon your attention, because I am sure it is a fact that has been very much overlooked, and to which sufficient importance has not been assigned. I have sufficiently explained it in my work on Asthma and elsewhere; and, therefore, I shall not now detain you by entering into its rationale.

Let me direct your attention to this patient's sputum. You may remember that I told you just now that his cough was at first quite dry, but that now some expectoration is appearing. Now, why should this man spit? He has had no bronchitis; he has 
had no pneumonia; his respiratory mucous tract has not been affected by any of those conditions which commonly give rise to fluxes and exudations from mucous surfaces. Where does the sputum come from, and why does it come? It comes from the only place from which mucous sputum can comefrom the bronchial mucous membrane ; and it comes because that membrane is congested. There are evidently two conditions in which mucous surfaces pour forth an inordinate secretion : one, active inflammation ; the other, passive congestion. In fact, any hyperæmia of a mucous surface, whether active or passive, relieves itself by an increase of the secretion of that surface. But there is, I think, this difference, that while the secretion from an inflamed mucous membrane is often highly purulent, that from a mucous surface merely congested is either not at all so, or very slightly; I am inclined to think, not at all. Now, if you look at this sputum you will see that it has no admixture of pus; that it consists of a grey gelatinous mucus, not opaque, and with no trace of yellow in it. It is very like the sputum of asthma, and is produced in the same way-poured out to relieve a state of congestion engendered by embarrassment of the pulmonary circulation. In pure asthma you have no inflammation of the bronchial mucous membrane, and yet it is very rare for an attack of asthma to pass off without the occurrence of this mucous exudation; never, I think, unless the attack is exceedingly transient. This is, in my opinion, an important distinction; and I believe that the presence or absence of pus will always enable us to diagnose whether the mucus is the result of an inflamed, or a merely loaded condition of the bronchial mucous membrane; at least, the presence of pus will always enable us to say that there is something more than mere congestion, although, in the very earliest stages of inflammation of the air-passages, the absence of pus will not prove that such inflammation does not exist.

And now, in conclusion, a word or two on treatment. What is to be done in a hopeless case like this, rapidly running to a fatal issue? How can we better this poor man's condition, incurable and unreachable as the organic foundation of it is ? In one way, and only in one way, that I know of. His disease is essentially mechanical, and in a mechanical and material direction we must aim at its relief. The machinery of his circulation is out of gear. Now, in the circulation of the blood, there are three things concerned-the active organ, the heart; the passive organs, the sanguiferous vessels; and the fluid to be circulated, the blood. In this case, the passive organs and the fluid to be circulated are as they should be ; the active organ is spoiled, and by this spoiling is no longer equal to its work. How, then, can we bring about some approximation between the work to be done and the power to do it? The heart we cannot reach ; and if we could, its derangement is essentially irremediable; the passive organs we cannot influence-we cannot shorten the distance the blood must reach, nor supplement the heart's deranged machinery by any fresh force. There is only one thing left; to diminish the quantity of the fluid to be circulated. We cannot raise the machine to its work; we must lower the work to the machine. If we can do this, we may hope, pro tanto, to disburden the embarrassed heart, and to relieve the choked and loaded ressels.
There are two ways in which this may be effected. Blood may be directly abstracted. And there is no doubt that immense relief would immediately follow venesection in such a case. But we know, from the rapidity with which some elements of the blood are restored, and the slowness with which others are, that such a quantitative reduction of the blood is speedily followed by a qualitative deterioration of that which is left. We do much better, then, if we can select what elements of the blood we will withdraw, and leave behind those which are the most valuable and of the slowest repair. Diuretics and hydrogogues enable us to do this. But I have found in these cases, direct diuretics peculiarly inoperative; I believe them to be almost worthless. When the mechanical embarrassment of the circulation is extreme, and the quantity of the urine greatly reduced (as it almost always is) by the slow renewal of the blood in the kidneys, you may give diuretics for ever and get no results. I believe the blood in the Malpighian capillaries is in a state almost of stasis, and, having parted with its water, is not promptly succeeded by relays of fresh blood with a plus quantity of water, as is the case in health. To give diuretics in such a state of things is beginning at the wrong end the renal circulation must be disembarrassed before the kidneys can filter away much water. IIydragogues then alone remain to us. Of these we have many ; our choice is large-jalap, salines, and a long list of purgatives. But by far the most powerful and satisfactory is elaterium. And I think, if I could show you some cases that have receutly come under my observation, you would be disposed to admit with me that the results attained by the administration of elaterium in cases of cardiac dropsy are among some of the most striking -I may say the most startling-triumphs of therapeutics. Floods of water are draughted away from the bowels, the dropsy vanishes, the breathing becomes unembarrassed, and the patient is restored, for a time, to a condition of very tolerable comfort. This is no exaggeration. Only about a month ago, I saw a very striking case. I was called to see a man at Camberwell, who was supposed to be, and really was, in extremis. I found him distended with dropsy; his legs like two Doric columns; and he had not been in bed for many nights, owing to the urgency of his dyspnœa. I heard, about a fortnight afterwards, that his improvement had been so rapid and so great under the use of elaterium, that he had returned to active business in town, and had resumed his employment as a traveller for one of the principal brewing firms in this city, and was at that time travelling in Northumberland. In fact, I am now never surprised at any amount of improvement that follows the use of elaterium in these cases. The only disadvantage that it possesses is, that the patients are often in a state of extreme exhaustion, and the action of the drug is frequently, in a high degree, depressing. T'o obviate this evil as much as possible, I allow sufficient time between the doses for the patient to recover himself, and, at the same time, freely support with stimulants and tonics. If the elaterium is given at long intervals the dose must, of course, be proportionately larger, and I find that the patient is less exhausted by a good dose every other day, than by smaller doses more frequently repeated. My plan is to give a third of a grain, or even half a 
grain, about five o'clock every other morning. By ten or eleven o'clock the whole thing is over; immense watery defluxions have taken place from the patient's bowels, leaving him wonderfully lightened and relieved, but much exhausted. (I generally begin, I ought to mention, with the sixth or eighth of a grain, and increase the dose as I find my patient will bear it. It is a dangerous thing to start with a large dose of elaterium at first; partly because different individuals tolerate it so differently, and partly on account of the unequal strength of the drug.) At the same time, I give some such mixture as the following, in a wineglass of water, every three or four hours :-

R Liquor. cinchon. (Battley) Mlxx; spirit. ammon. arom. 3 ss; ætheris sulph. co. 3 ss. M.

Under the influence of this, with the aid of the intervening recruiting day, patients will bear the quantity of elaterium I have mentioned for an indefinite time, and even gain strength under it, or, rather, in spite of it. A fortnight of such treatment is often sufficient entirely to transform the patient's condition. One important result of this treatment is that, with the disappearance of the rest of the dropsy, the œdema of the lungs also vanishes; thus, one source of the dyspnœa is removed, and, by the free admission of air into those portions of the lungs previously occupied by the serous infiltration, the asphyxial element of the pulmonary blood-stasis is cleared away, and one additional source of heart embarrassment removed. I think this is one of the most important results of this hydragogue treatment : the other results relieve the breathing indirectly, this directly. It is a most interesting thing to watch, day by day, under these circumstances, the gradual descent of the signs of the œdema at the back of the lung till not a trace of it remains.

Now, this treatment we have tried with this poor man, with decided but, unfortunately, partial benefit. Under its use, the dropsy, which was previously gaining upon him, had, in a week, almost ranished; but the dyspnœa and other urgent symptoms were not a whit the better. At the end of that time, from the vomiting and great stomach disturbance that the drug produced, a circumstance for which, in the administration of elaterium, we must be prepared, we were obliged to remit it, and its resumption has less completely succeeded in controlling the dropsy. Why it is that, in this case, the dyspncea and other symptoms have been so little relieved by the reduction of the volume of the blood, and its immediate results, I am as unable to explain as I am why the respiratory symptoms should be altogether so disproportionately developed.

A question might arise in the minds of many, whether, since sleeplessness is one of the principal and most distressing symptoms, sedatives might not be given with advantage. To give sedatives in such a case would be the refinement of cruelty. What keeps this poor man awake is not a want of tendency to sleep, but a condition that makes sleep impossible. Relieve him of his orthopnca and he would be asleep in ten seconds, and so dead asleep that it would take a great deal to rouse him, like a half-asphyxiated child on whom tracheotomy had just been performed. His great struggle, as it is, is the struggle between sleep and life; with opium thrown into the scale of sleep the struggle for life would only be so much the harder. In one way, and only in one way, would opium give him ease : the narcotic of opium, added to the narcotic of the carbonic acid already circulating in his veins, might accelerate by some hours, or even days, the final coma, and make him sooner sleep the sleep of death. But the euthanasia that is purchased by anticipating the natural process of death, comes very near to homicide, and is an alternative that few would adopt.

\section{OPriginal Communitations.}

\section{CASE OF DISLOCATION OF THE SHOULDER- JOINT, AND OF FRACTURE THROUGH THE \\ NECK OF THE HUMERUS, OCCURRING DURING AN EPILEPTIC SEIZURE.}

By Robert DunN, F.R.C.S.Eng.

ON the afternoon of Saturday, September 28th, 1861, was hastily called to Mr. B., a young gentleman residing at 10, Norfolk Street, who had been taken in a fit of epilepsy, to which he was subject. In my absence, my son, R. W. Dunn, promptly and at once attended. Before, however, he could cross the street to him, the convulsive paroxysm had subsided. $\mathrm{He}$ found $\mathrm{Mr}$. B. in his bedroom, sitting on a chair before the window, beside the washhand-stand and the wooden horse, on which were placed the towels in use. Mr. B. com: plained of excessive pain on the anterior part of the left shoulder-joint, and that his arm was powerless. My son was told by his brother that he had been subject to epileptic fits for some years, and had very recentlyonly a day or two previously-come up expressly from Scotland to have the benefit of Dr. Brown-Séquard's advice. He further informed him that, twice in the course of the current month, during the epileptic seizure, he had dislocated his left shoulder-joint. On the present occasion, while standing before the window, he was seized with the fit, and would have fallen on the. floor, if he had not saved him. He thought it possible: that his shoulder might have come against the wooden horse in the struggle, but not with violence; nor was there, on examination, any ap pearance of bruise or contusion about the joint. My son immediately saw that the shoulder had again been dislocated forwards, the head of the humerus being visibly prominent under the pectoral muscle; but this time the dislocation was attended with an excessive degree of pain in the part, which had not before been experienced. He at once set about the reduction of the dislocation in the usual way. In his attempt to do this, the head of the bone was moved from its position; and, with this change of posture, the excessive pain was relieved; but it did not slip into the glenoid cavity.

Being satisfied that there was some unusual complication in the case, and as the pain was relieved, he made no further attempt at reduction, but waited for me. On our carefully examining the shoulder together, I detected crepitation through the neck of the humerus. As a medical friend of the patient, an army surgeon from Scotland, was, by appointment, about this time expected to call upon him, we did not attempt anything further until his arrival, when, after consulting together, another effort was made, chiefly by manipulation about the head of the bone and slight extension; but our united efforts failed to reduce the dislocation. I then suggested to the friends that we should have the assistance of Mr. Fergusson, to which they gave a ready assent, and he was immediately sent for; but he was 